\title{
OUTCOME OF TOCILIZUMAB THERAPY IN CHILDREN WITH SYSTEMIC-ONSET JUVENILE IDIOPATHIC ARTHRITIS
}

\author{
S. Balameena ${ }^{1}$, R. Ravichandran ${ }^{2}$
}

${ }^{1}$ Senior Assistant Professor, Department of Rheumatology, MMC, Chennai, Tamilnadu, India.

${ }^{2}$ Director and Professor, Department of Rheumatology, MMC, Chennai, Tamilnadu, India.

ABSTRACT
BACKGROUND
Globally, 3 lakh children suffer with idiopathic arthritis amongst which systemic is about $10 \%$ to $25 \%$. The
severe, mortality is about $2 \%-4 \%$ and hence different treatment protocols are used.
Aims and Objectives- To find out the clinical and laboratory outcome of Tocilizumab in children with SOJIA.

\section{MATERIALS AND METHODS}

It is a descriptive study.

Methods- 24 children of above 3 years to sixteen, who satisfied ILAR criteria for systemic onset, who were already on DMARDs and attended the rheumatic care centre at Rajiv Gandhi Govt. General Hospital were included between March 2014 and March 2017. Other types of JIA and very sick children were excluded. Tocilizumab $8 \mathrm{mg} / \mathrm{kg}$ was given every month and the clinical and laboratory assessment, ACR disease activity core set index were done at baseline and at 6 months.

\section{RESULTS}

Amongst 24 children, there were 3 dropouts. Tocilizumab was given in 12 doses in 6, 6 doses in 7, 7 doses in 4 and 3 doses in 4 . Male: Female seen as 16: 5 or 3.2: 1 . Mean age in the cohort was $9.1 \pm 0.729$. Mean disease duration was $4.09 \pm 0.43$. Investigation showed Mean ESR $69.05 \pm 3.78$ and $38.09 \pm 2.52$; Mean CRP $25.71 \pm 4.06$ and $5.43 \pm 1.30$; Mean WBC $18.92 \pm 2.66$ and $9.54 \pm 0.56$; Mean platelet $6.27 \pm 3.7$ and $4.68 \pm 0.189$ before and after Tocilizumab therapy.

\section{CONCLUSION}

Tocilizumab therapy for systemic JIA reduces systemic activity to large extent along with articular inflammation, seen as drop in CRP and ESR and platelet with improvement in ACR 30\% in this study.

\section{KEY WORDS}

JIA, SOJIA and Tocilizumab.

HOW TO CITE THIS ARTICLE: Balameena S, Ravichandran R. Outcome of tocilizumab therapy in children with systemic-onset juvenile idiopathic arthritis. J. Evolution Med. Dent. Sci. 2018;7(23):2746-2748, DOI: 10.14260/jemds/2018/620

\section{BACKGROUND}

Globally, 3 lakh children suffer with idiopathic arthritis, amongst systemic is about $10 \%$ to $25 \%$. The morbidity of arthritis is severe and mortality is about $2 \%-4 \%$ and hence different treatment protocols are available.

JIA is seen in 150 - 200 children in 1 lakh population around the world, which is the commonest occurring rheumatological disorder. Amongst JIA, SOJIA forms 10\%$25 \%$. It affects age group of 0 - 5 years. It affects male and female children equally.

In 1987, Sir George Alfred still described this condition as it presents with systemic features like fever in $80 \%$, salmonpink rash affecting the trunk usually evanescent in $60 \%$, lymphadenopathy in 32\%, hepatosplenomegaly in $50 \%$, serositis in $10 \%$ and arthritis in $60 \%$, rarely seizures, cholecystitis and uveitis. ${ }^{[1]}$

Goldberg described 2 out of 11 patients in cohort with myocarditis, pericarditis and pleuritis in 4 out of 11 along

'Financial or Other Competing Interest': None.

Submission 02-01-2018, Peer Review 21-05-2018,

Acceptance 28-05-2018, Published 04-06-2018.

Corresponding Author:

Dr. S. Balameena

18, 1/708, $1^{\text {st }}$ Street,

MCN Nagar, Thoraipakkam,

Chennai-97, Tamilnadu, India.

E-mail: balameenakumar@gmail.com

DOI: $10.14260 /$ jemds $/ 2018 / 620$

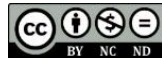

with raise of acute phase reactants with transaminitis. It carries severe morbidity of the joints and mortality when persistent systemic features leading to macrophage activation syndrome in some cases.

Immunopathogenesis involves interplay of IL1 and IL6 mediated inflammation of macrophages along with reactive thrombocytosis and anaemia. Hepcidin which prevents reflux of iron from monocytes and liver cells into plasma causes low serum iron and leads to anaemia. Other interleukins MRP8 and 14, MMIF, IL-18 play a role during active inflammation. IL-4 1098 T/G Polymorphisms. MEFV gene mutations, pyrin dysfunction are implicated in initiation of this disease.[1]

\section{Complication}

Children develop malnutrition due to poor intake leading to short stature, osteoporosis and school absenteeism. Macrophage activation syndrome due to disease activity and is also seen in viral infections. In long-term disease activity, amyloidosis reported by Immonen et al at 5 yrs. was $88 \%$ and at 10 yrs. was $75 \%$.

Due to drugs and immunosuppression children develop infections, Cushingoid features, osteoporosis and anaemia.

\section{Differential Diagnosis}

Include familial HLH and periodic syndrome. Synthetic conventional DMARDs were useful in JIA, but when resistant to these drugs anti-cytokine therapy is given. ${ }^{[2]}$ 
Tocilizumab is an IL-6 monoclonal antibody, which is used to reduce the systemic activity in severe cases and also steroid sparing drugs.[3,4] Tocilizumab was introduced in Japan for Castleman's disease in 2005 and then approved for SOJIA in 2008. Same drug was approved in Europe in 2009 and by FDA in 2011 for SOJIA in United States.

Minor side effects being mild URTI, nasopharyngitis in 5\% and headaches and diarrhoea in 3\% which recovers. Serious adverse event seen as bacterial pneumonias, varicella infection, otitis media and septicaemia.

In tender study using Tocilizumab $8 \mathrm{mg} / \mathrm{kg}$ in 2 phases, preliminary period for 12 weeks and extended period of 104 weeks ( 2 yrs.) showed the drug was efficacious. Another ongoing study for 5 years in 17 countries included 12 weeks period and primary endpoint at 12 weeks was absence of fever. They found $85 \%$ of children had ACR 30 improvement compared to placebo $24 \%$. They found $71 \%$ of children had ACR 70 improvement compared to $8 \%$ in placebo. Mild side effects of tocilizumab noticed were URTI, headache, diarrhoea and nasopharyngitis in 5\%.[5,6]

Aims and Objectives: To find out the clinical and laboratory outcome of Tocilizumab in children with SOJIA.

\section{MATERIALS AND METHODS}

It is a descriptive study.

\section{Methods \\ Inclusion Criteria}

24 children of above 3 years to sixteen, who satisfied ILAR criteria for systemic onset, who were already on DMARDs and attended the rheumatic care centre at Rajiv Gandhi Govt. General Hospital were included between March 2014 and March 2017.

\section{Exclusion Criteria}

Other types of JIA and very sick children were excluded.

Tocilizumab $8 \mathrm{mg} / \mathrm{kg}$ was given to the children for every month along with methotrexate and prednisolone $5 \mathrm{mg}$ /day. Physician global assessment of disease activity, patient/parent global assessment of well-being, functional disability, no. of active joints, no. of joints with limitation and ESR assessed. For paediatric ACR, 30 disease activity core set index - 30\% improvement of 3 out of 6 variables and not $30 \%$ worsening in any one is taken into index calculation. Periodical clinical and laboratory assessment was done at baseline and at 6 months and at 1 year.

The different laboratory parameters were analysed along with clinical index before and after tocilizumab therapy. The laboratory outcomes ESR, CRP along with the clinical paediatric ACR 30\% before and after tocilizumab therapy were analysed in descriptive manner and depicted as graphical representation.

\section{RESULTS}

Amongst 24 children, 3 dropouts were seen. Tocilizumab was given in 12 doses in 6, 6 doses in 7, 7 doses in 4 and 3 doses in 4. Male: Female seen as 16: 5 or 3.2: 1. Mean age in the cohort was $9.1 \pm 0.729$. Mean disease duration was $4.09 \pm$ 0.43 .

Constitutional features include fever in all the patients $100 \%$, rash in $85.7 \%$ and weight loss in $52.4 \%$ as shown in Figure 1.

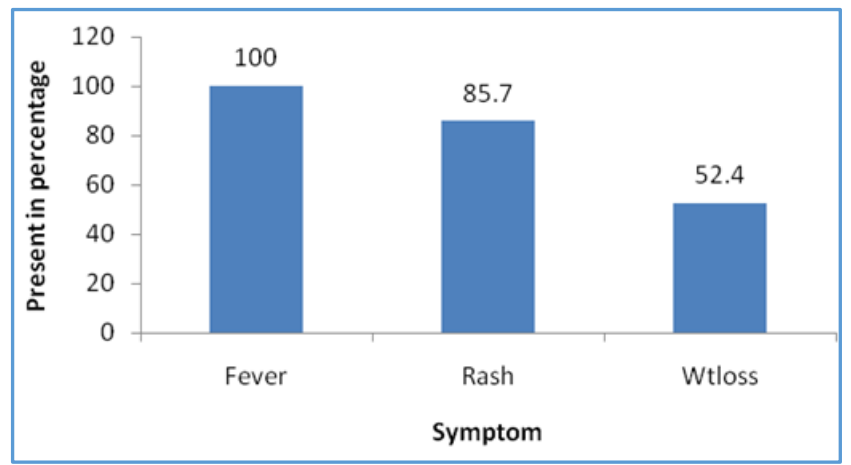

Figure 1. Clinical Feature

Joints involved were both small and large joints in the course of the illness and was seen in all patients $100 \%$ and neck in $66.7 \%$ and hip involved in $42.9 \%$ as shown in Figure 2.

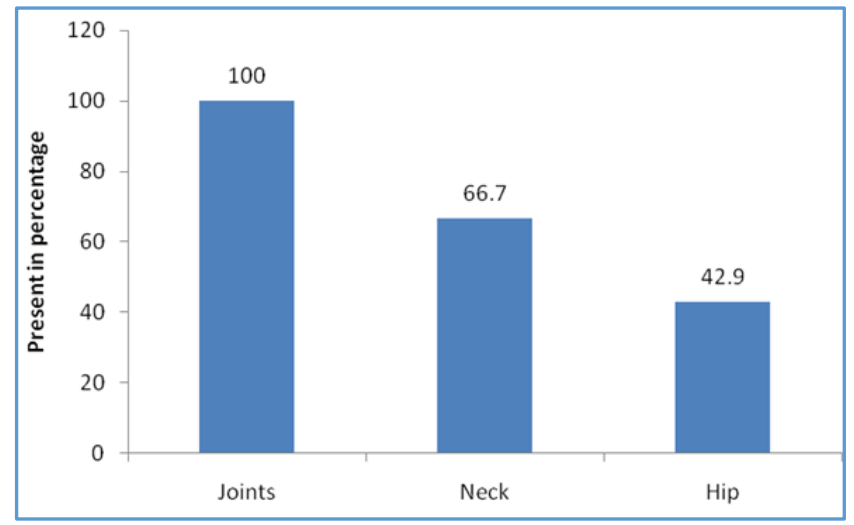

Figure 2. Joint Involvement

Investigation showed Mean ESR of $69.05 \pm 3.78$ and $38.09 \pm 2.52$ (Fig. 3), Mean CRP of $25.71 \pm 4.06$ and $5.43 \pm 1.30$ (Fig. 4), Mean platelet of $6.27 \pm 3.7$ and $4.68 \pm 0.189$ (Fig. 5) before and after Tocilizumab.

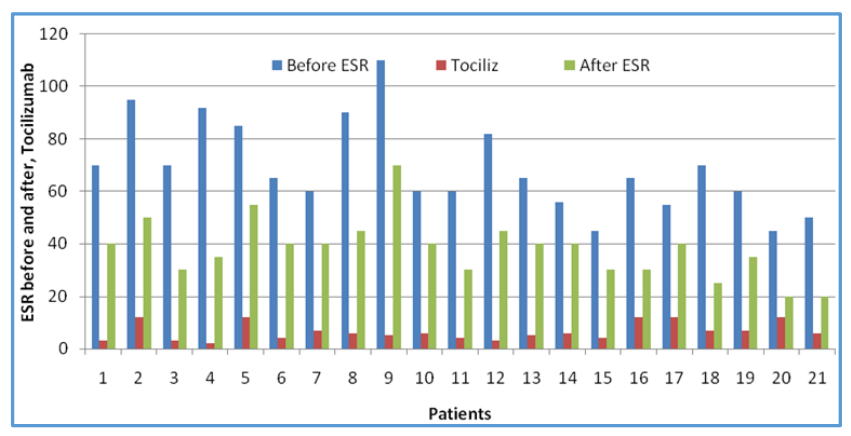

Figure 3. ESR before and after Tocilizumab

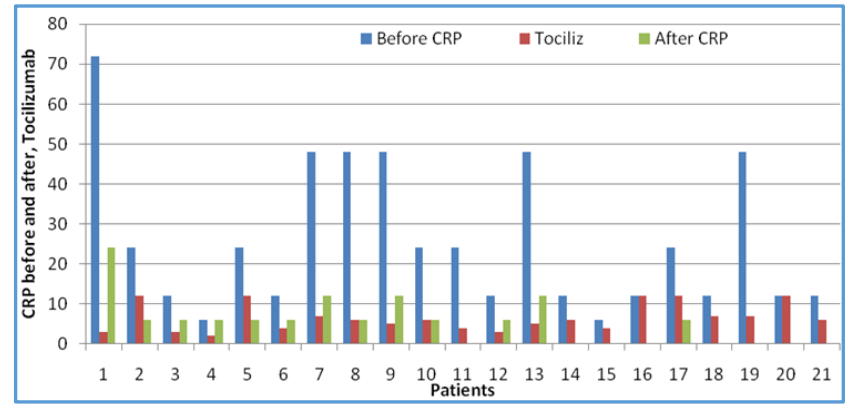

Figure 4. CRP before and after Tocilizumab 


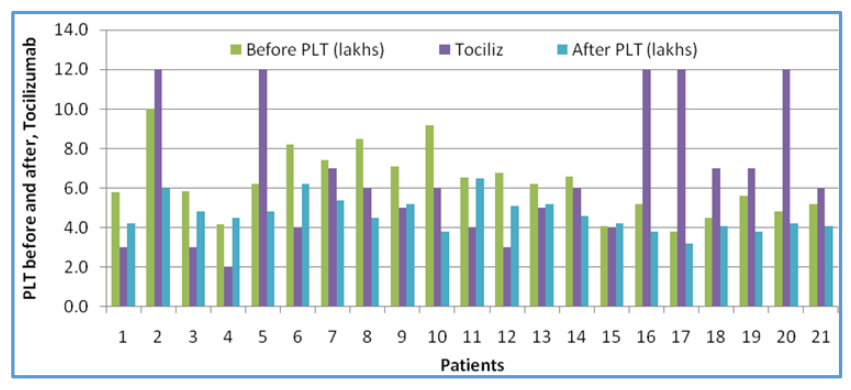

Figure 5. Platelet Count before and after Tocilizumab

In this study, Mean WBC was $18.92 \pm 2.66$ and $9.54 \pm 0.56$ and it also shows encouraging results with drug intervention.

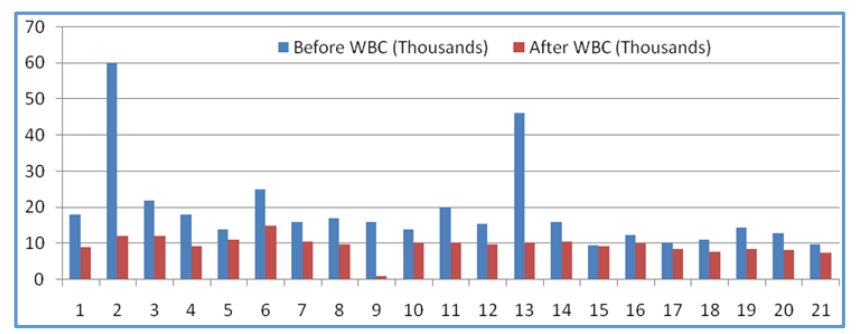

Figure 6. WBC before and after Tocilizumab

Paediatric ACR 30\% improvement core set index also shows positive change with Tocilizumab Fig. 7.

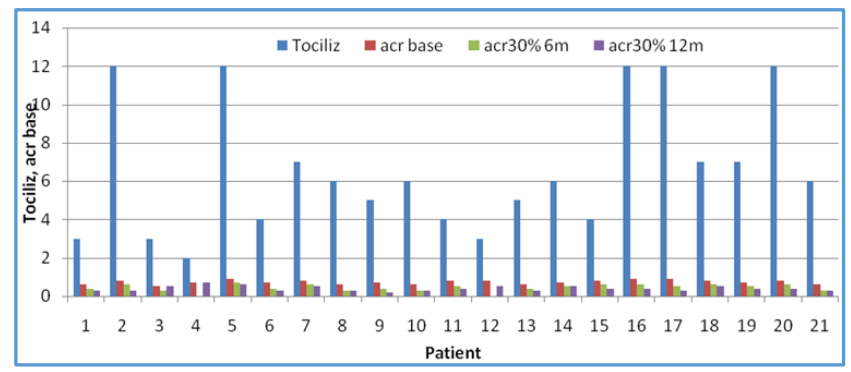

Figure 7. Paediatric ACR 30\% Baseline and ACR 30\% Improvement after Tocilizumab

\section{DISCUSSION}

Two-year results of the TENDER study demonstrated sustained efficacy of TCZ. In terms of SOJIA, improvement with TCZ occurred early and was maintained over time. About $60 \%$ of patients treated with TCZ for 104 weeks were able to discontinue oral corticosteroids and $37 \%$ of patients achieved inactive disease status by week 104 . No new safety signals emerged during longer term treatment.

Common AEs included infections, neutropenia and increases in alanine transaminase level's response to TCZ was not affected by disease duration, presence/ absence of active systemic features at baseline, number of active joints at baseline, baseline oral corticosteroid dose or concomitant use of MTX and prior treatment with biologics.

In George Fredrick study fever was seen in $80 \%$, but in our cohort it was present in all $100 \%$. In Behren et al study rash was seen in $81 \%$, but in our study it was $85.7 \%$. Joints present was $100 \%$ in our study, but in Behren et al it was $60 \%$ only. In this study, systemic-onset fever pattern in children is one of the characteristic feature which is the cause for leukocytosis and thrombocytosis. This increased cell counts account for the severe inflammation and disease activity. After Tocilizumab treatment systemic features responded well and there is a marked reduction in cell counts, total WBC and platelets.

The acute phase reactants, ESR and CRP showed reduction after tocilizumab treatment. Paediatric ACR of $30 \%$ improvement in disease activity was seen in 6 months with Tocilizumab therapy.

There was no association seen with disease duration and Tocilizumab therapy, which was also seen in TENDER study.

\section{CONCLUSION}

TCZ was effective across multiple baseline characteristics. TCZ treatment resulted in a rapid and sustained reduction of inflammation associated with SOJIA.

Tocilizumab therapy for systemic JIA reduces systemic activity to large extent and articular inflammation seen by the drop in CRP and ESR and platelet with improvement in ACR $30 \%$ in this study.

\section{Limitation}

As this is a small cohort, hence larger data will give more valuable information.

\section{REFERENCES}

[1] Gurion R, Lehman TJA, Moorthy LN. Systemic arthritis in children, a review of clinical presentation and treatment. Article ID: 271569, International Journal of Inflammation 2012: p. 16.

[2] Beukelman T. Treatment advances in systemic juvenile idiopathic arthritis. F1000 Prime Reports 2014;6:21.

[3] De Benedetti F, Brunner H, Allen R, et al. Tocilizumab is efficacious in patients with SOJIA across baseline demographic and disease characteristics and prior/baseline treatments: 52-week data from a phase 3 clinical trial. Arthritis and Rheumatism 2011a;63(Suppl 1):2621.

[4] Singh-Grewal D, Schneider R, Bayer N, et al. Predictors of disease course and remission in SOJIA: significance of early clinical and laboratory features. Arthritis Rheum 2006;54(5):1595-601.

[5] Yokota S, Imagawa $\mathrm{T}$, Mori $\mathrm{M}$, et al. Long term treatment of SOJIA with tocilizumab: results of an open-label extension study in Japan. Ann Rheum Dis 2013;72(4):627-8.

[6] Yokota S, Imagawa T, Mori M, et al. Efficacy and safety of tocilizumab in patients with SOJIA: a randomized double-blind, placebo-controlled withdrawal phase III trial. Lancet 2008;371(9617):998-1006. 